\title{
Zebrafish mutants of the neuromuscular junction: swimming in the gene pool
}

\author{
Eriko Daikoku $^{1} \cdot$ Masahisa Saito $^{1} \cdot$ Fumihito Ono $^{1,2}$
}

Received: 22 January 2015/ Accepted: 28 February 2015/Published online: 18 March 2015

(C) The Author(s) 2015. This article is published with open access at Springerlink.com

\begin{abstract}
This review provides an overview of zebrafish mutants with dysfunctional acetylcholine receptors or related proteins at the neuromuscular junction (NMJ). The NMJ, which has served as the classical model of the chemical synapse, uses acetylcholine as the neurotransmitter, and mutations of proteins involved in the signaling cascade lead to a variety of behavioral phenotypes. Mutants isolated after random chemical mutagenesis screening are summarized, and advances in the field resulting from these mutants are discussed.
\end{abstract}

Keywords Synapse $\cdot$ Zebrafish $\cdot$ Acetylcholine receptor · Mutant

The zebrafish is an animal model for biomedical research which has continuously increased in popularity over the past several decades. Its unique features make this small tropical fish an excellent model in a wide variety of biomedical fields. In addition to conventional whole-cell patch clamp, a plethora of genetic tools developed over the years, in combination with the transparency of the embryonic and larval stages, render zebrafish particularly amenable to optics-based physiological techniques, for example optogenetics [1] or calcium imaging [2, 3]. In this mini review we summarize advances in the study of synapses in zebrafish, focusing on the neuromuscular junctions (NMJs).

Fumihito Ono

onof@art.osaka-med.ac.jp

1 Department of Physiology, Osaka Medical College, Takatsuki 569-8686, Japan

2 Laboratory of Molecular Physiology, NIAAA, NIH, Bethesda, MD 20892, USA

\section{Behavior mutants isolated by random mutagenesis}

When random mutagenesis by use of $N$-ethyl- $N$-nitrosourea (ENU) was first applied to zebrafish in the 1990s [4], most of the mutants isolated by large-scale screening were identified on the basis of characteristic morphological abnormalities at the embryonic stage. In addition to these morphological mutants, a group of mutants with abnormal behavior was also isolated [5]. Mutants in this group were relatively slow to attract attention compared with the morphological or developmental phenotypes. Symptoms of these mutants ranged from total paralysis to reduced motility and circular swimming. Subsequent identification, one by one, of the genes underlying these behavioral phenotypes shed light on the neural system, furnishing new information on the function of the genes underpinning the motor system.

\section{Mutants of AChR subunits}

Many of the locomotion mutations mapped to genes coding for synaptic proteins in the NMJs. On the presynaptic side, synaptic vesicles containing acetylcholine are released from motor neuron endings in response to membrane excitation. Zebrafish mutants of pre-synaptic proteins, for example NSF [6] or the voltage-sensitive calcium channel [7], were identified and analyzed; these will not be discussed in this review. On the post-synaptic side, acetylcholine receptors (AChRs) receive acetylcholine released from the motor neuron endings and conduct inward currents through the open pore, leading to a cascade of events that culminates in the contraction of the muscle fiber. AChRs at NMJs comprise of five subunits (thus called pentamers): two $\alpha 1 \mathrm{~s}$, one $\beta 1$, one $\delta$, and one $\varepsilon$ or $\gamma$, with 
the $\varepsilon$ and $\gamma$ subunits being interchangeable. The $\gamma$ subunit is expressed early in development and, in mammals and fish, is replaced by the $\varepsilon$ subunit as the animal matures $[8,9]$. A notable exception to this long-held view, which will be discussed in detail below [10,11] (Fig. 1), is the recent finding that NMJs in some types of zebrafish muscle fiber have pentamers that have neither the $\varepsilon$ nor $\gamma$ subunit but, instead, have two $\delta$ subunits.

Two null mutants were identified in zebrafish AChR subunits (Fig. 2). One mutant, nic [12], mapped to the $\alpha 1$ subunit and is a result of a splicing error that blocks the synthesis of the $\alpha 1$ subunit. Another mutant, sofa potato [13], mapped to the $\delta$ subunit. In contrast with nic, a fulllength $\delta$ subunit is synthesized. However a conserved leucine residue is changed to proline near the $\mathrm{N}$-terminus, which blocks the assembly of pentamers. In nic and sofa potato, AChR pentamers fail to form and the remaining normal subunits are retained in the endoplasmic reticulum [14]. These null mutants are completely paralyzed until their death approximately 7 days post-fertilization (dpf), but can be rescued by introducing a normal copy of the mutant subunit $[13,15]$. The rescued null mutant can grow to adulthood and breed normally [15]. The subunit used for rescue can also be fused to a fluorescent molecule in the long intracellular loop, which makes the introduced protein visible.

The absence of a $\beta 1$ mutant isolated from random mutagenesis screening is puzzling. The $\beta 1$ subunit, a structural subunit which does not contribute to binding of the ligand, is absolutely necessary to form AChR pentamers [10, 16]. Therefore a null mutant of the $\beta 1$ subunit will lack AChRs in the NMJ and, hence, detection by phenotype-based screening is expected to be straightforward. One possible

Adult
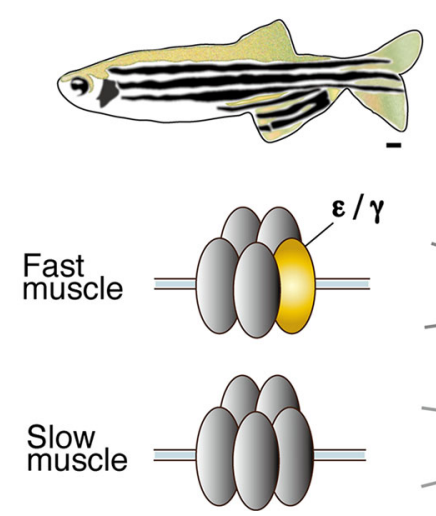

Fig. 1 Schematic diagram of the zebrafish NMJ. In larvae, AChR clusters are located at the edge of slow muscle fibers and form chevron-shaped lines at the boundary of body segments, whereas those in fast muscle fibers are observed as round, punctate dots distant from the edges. AChRs in slow and fast fibers have subunits of different composition. Scale $1 \mathrm{~mm}$ explanation is duplication of the $\beta 1$ gene in the zebrafish genome. In teleost genomes, some genes have duplicated copies resulting from fish-specific genome-wide duplication (FSGD) supposed to have occurred between 335 and 404 million years ago [17]. Two duplicated copies in zebrafish, $\beta 1 \mathrm{a}$ and $\beta 1 \mathrm{~b}$, encode subunits homologous to the mammalian $\beta 1$. However, when expressed in Xenopus oocytes with other subunits, $\beta 1$ a did not lead to robust expression of functional receptors [10, 18]. Although it is possible the $\beta 1$ a subunit is functional in vivo, There are no convincing data showing that $\beta 1 \mathrm{a}$ can compensate for $\beta 1 \mathrm{~b}$ when the latter is knocked out.

\section{Point mutations of $\mathrm{AChR}$ subunits cause striking phenotypes}

A merit of random mutagenesis compared with reverse genetics is that random point mutations often lead to unexpected phenotypes and reveal previously unrecognized aspects of protein function. In zebrafish AChRs, the twister mutant [19] and the love sofa mutant [11] are such examples. The twister mutant has a point mutation in the $\alpha 1$ subunit whereas the love sofa mutant has a point mutation in the $\delta$ subunit (Fig. 2).

The twister mutation mapped to a leucine residue (L258P) in the M2 trans-membrane region (Figs. 2, 3). AChRs containing the $\alpha 1$ subunit with the twister mutation have longer channel openings leading to a stronger, prolonged muscle contraction [20]. Fish homozygous for the twister mutation are embryonic lethal whereas heterozygotes for twister result in phenotypes linked with prolonged

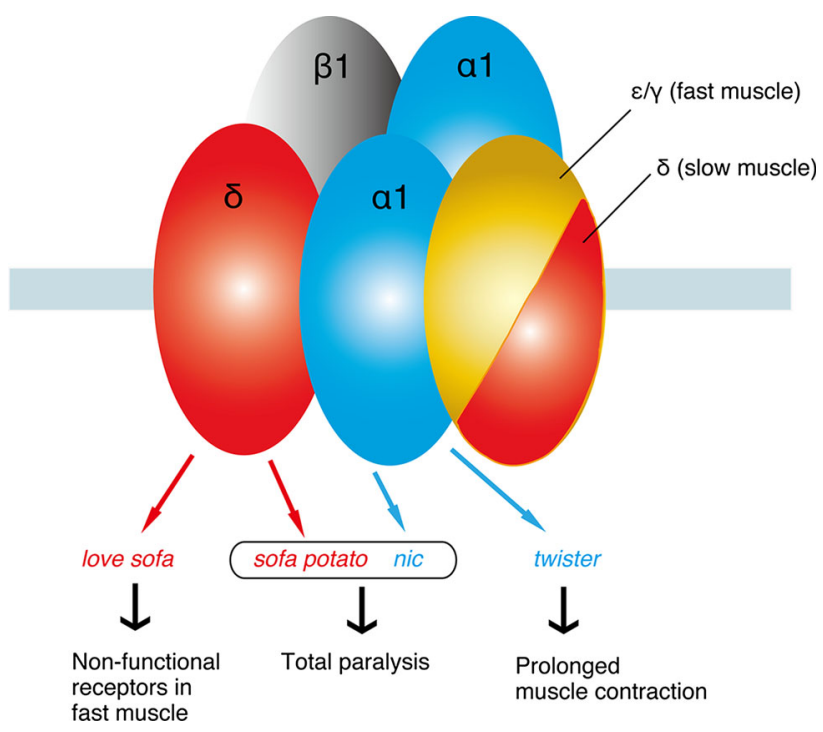

Fig. 2 Zebrafish mutants of AChRs. Subunits harboring the mutation, their names, and the phenotypes are shown 


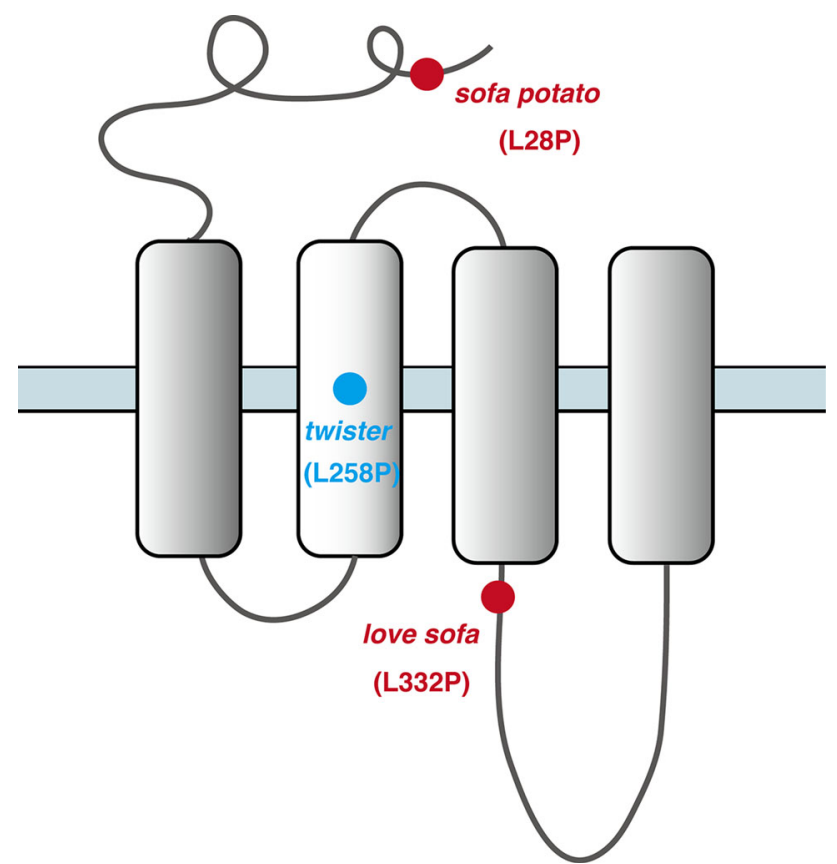

Fig. 3 Locations of mutations in twister, sofa potato, and love sofa are indicated relative to the trans-membrane regions of the $\alpha$ (for twister) or $\delta$ (for sofa potato and love sofa) subunit. In each mutant, a leucine residue was changed to proline

muscle contraction. Interestingly this phenotype can only be observed during early development [19], and heterozygotes recover from their behavioral defect as they age, which coincides with a change of synaptic current kinetics. This recovery is based on the developmental subunit switch from $\lambda$ to $\varepsilon$ [9].

The love sofa mutant, caused by a point mutation in the $\delta$ subunit, gives rise to a peculiar phenotype (Fig. 2). Similar to mammals, zebrafish skeletal muscle contains two fiber types, i.e. slow muscle fibers and fast muscle fibers (also called type I and type II fibers, respectively, in mammalian muscles) [21]. Fish homozygous for the love sofa mutation only form functional synapses in slow muscle fibers whereas fast muscle fibers have non-functional receptors that do not conduct currents in response to released acetylcholine. Although mutations in some muscle genes skew the proportion of fiber types as the skeletal system adapts to muscle degeneration [22], to the best of our knowledge, no mutation other than love sofa causes fiber type-specific phenotypes restricted to the synapse. This unexpected effect of the mutation arises from the different composition of subunits in the two types of fiber [11]. Slow muscle fibers lack $\varepsilon / \gamma$ subunits and have two $\delta$ s instead, which also underlies the different kinetics of AChRs [10] (Figs. 1, 2). This is, as stated above, a deviation from a traditional view of AChR subunit composition, in which $\varepsilon$ or $\gamma$ was believed to be a necessary component of AChRs at NMJs in vivo. The love sofa mutation is located in the basal region of the cytoplasmic loop between the third and fourth trans-membrane regions (Fig. 3), and is likely to affect the global structure of the AChR pentamer, rendering the subunit combination found in fast muscle fibers non-functional yet sparing that of the slow muscle fibers. These findings show that zebrafish is a useful model to investigate the difference between the NMJ of slow and fast muscle fibers at the molecular level.

\section{Rapsyn regulates $\mathrm{AChR}$ in a reciprocal manner}

Rapsyn is a myristoylated cytoplasmic protein expressed in muscle cells that interacts directly with AChRs and is important in regulation of AChR clustering [23]. On the basis of a behavioral phenotype, a rapsyn mutant called twitch once has also been identified in zebrafish. This mutant has an escape response upon touch [24]. However the muscle contractions weaken and the swimming stops after a few tail bends. This phenotype is reminiscent of the muscle fatigue observed in human myasthenic patients and, interestingly, mutations in the human rapsyn gene cause the congenital myasthenic syndrome [25]. The twitch once mutant harbors a mutation in the tetratricopeptide repeat domain of the rapsyn protein and does not form AChR clusters at the synapse [24]. Instead AChRs are distributed diffusely on the plasma membrane.

Although rapsyn interacts with several proteins [26], its interaction with AChRs is best characterized and central to its function. Although rapsyn was originally identified as a factor that binds to AChRs at a 1:1 ratio, later analysis indicated that the stoichiometry is less rigid [27]. Analysis of the interaction of rapsyn with AChR subunits by use of conventional biochemical techniques proved onerous, and over the years several laboratories have used elegant techniques to unravel the mechanism of protein interaction $[28,29]$. Notably, by use of chimeras of CD4 and AChR subunits, phosphorylation of the AChR $\beta$ subunit was shown to intensify the binding of rapsyn to AChRs [30].

Rapsyn was initially believed to regulate AChR localization without any input from the AChR. More recent studies using the AChR null mutant (sofa potato) and cellline systems revealed that the regulation is actually reciprocal [31, 32]. Rapsyn cannot localize to the synapse correctly in the absence of AChRs [13]. In particular, analysis of rapsyn in sofa potato revealed that rapsyn cannot reach the plasma membrane without AChRs and is retained in the Golgi apparatus [14]. When normal AChRs were introduced in sofa potato, the transport of rapsyn to the plasma membrane was restored. This effect of AChR on rapsyn was highly specific: the expression of a different AChR 
molecule, normally expressed in the brain [33], failed to restore rapsyn localization [14].

\section{Conclusion}

Studies of mice and humans have revealed molecules involved in the development of post-synaptic structure at the NMJ, for example muscle-specific kinase (MuSK), dok-7, or Lrp4 [34-36]. Zebrafish mutants harboring mutations in these genes have also been identified. For example, a mutant of MuSK, unplugged, has defects in AChR distribution and in axon pathfinding [37, 38]. Further analysis of this MuSK zebrafish mutant revealed regulation by Wnt signals [39].

The zebrafish mutants discussed in this review provided valuable insights into the pathophysiology of some neurological disorders. Sofa potato and twitch once had mutations in genes later identified to cause human diseases $[13,24,25,40]$, and physiological study of twister indicated unappreciated benefits of subunit switch in myasthenic cases [9].

One notable feature of the zebrafish NMJ is its accessibility for optical, time-lapse studies in vivo. Ex-utero development combined with genetically introduced fluorescent molecules enable studies in which synapse formation can be observed in its entirety, both spatially and temporally [14, 41, 42]. Studies utilizing these unique approaches complement studies using human patients or mammalian animal models. Recent advances in genomeediting techniques make reverse genetics applicable to zebrafish [43]. Application of these new techniques will render the zebrafish system an even more useful model, which will continue to clarify the formation and function of synapses.

Acknowledgments We thank Dr Henry Puhl for critical reading of the manuscript. We thank Ms Kasumi Minamimoto and Ms Chika Murakami for their help with preparation of the figures. This work was supported by the intramural program at NIAAA.

Conflict of interest We declare no conflict of interest.

Open Access This article is distributed under the terms of the Creative Commons Attribution License which permits any use, distribution, and reproduction in any medium, provided the original author(s) and the source are credited.

\section{References}

1. Wyart C, Bene FD, Warp E et al (2009) Optogenetic dissection of a behavioural module in the vertebrate spinal cord. Nature 461:407-410
2. Ahrens MB, Li JM, Orger MB et al (2012) Brain-wide neuronal dynamics during motor adaptation in zebrafish. Nature 485:471-477

3. Ikeda H, Delargy AH, Yokogawa T et al (2013) Intrinsic properties of larval zebrafish neurons in ethanol. PLoS One 8:e63318

4. Haffter P, Granato M, Brand M et al (1996) The identification of genes with unique and essential functions in the development of the zebrafish, Danio rerio. Development 123:1-36

5. Granato M, van Eeden FJ, Schach U et al (1996) Genes controlling and mediating locomotion behavior of the zebrafish embryo and larva. Development 123:399-413

6. Woods IG, Lyons DA, Voas MG et al (2006) nsf is essential for organization of myelinated axons in zebrafish. Curr Biol $16: 636-648$

7. Wen H, Linhoff MW, Hubbard JM et al (2013) Zebrafish calls for reinterpretation for the roles of $\mathrm{P} / \mathrm{Q}$ calcium channels in neuromuscular transmission. J Neurosci 33:7384-7392

8. Mishina M, Takai T, Imoto K et al (1986) Molecular distinction between fetal and adult forms of muscle acetylcholine receptor. Nature 321:406-411

9. Walogorsky M, Mongeon R, Wen H et al (2012) Zebrafish model for congenital myasthenic syndrome reveals mechanisms causal to developmental recovery. Proc Natl Acad Sci USA 109:17711-17716

10. Mongeon R, Walogorsky M, Urban J et al (2011) An acetylcholine receptor lacking both $\gamma$ and $\varepsilon$ subunits mediates transmission in zebrafish slow muscle synapses. J Gen Physiol 138:353-366

11. Park JY, Mott M, Williams T et al (2014) A single mutation in the acetylcholine receptor $\delta$-subunit causes distinct effects in two types of neuromuscular synapses. J Neurosci 34:10211-10218

12. Sepich DS, Wegner J, O'Shea S, Westerfield M (1998) An altered intron inhibits synthesis of the acetylcholine receptor alpha-subunit in the paralyzed zebrafish mutant nic1. Genetics 148:361-372

13. Ono F, Mandel G, Brehm P (2004) Acetylcholine receptors direct rapsyn clusters to the neuromuscular synapse in zebrafish. J Neurosci 24:5475-5481

14. Park JY, Ikeda H, Ikenaga T, Ono F (2012) Acetylcholine receptors enable the transport of rapsyn from the golgi complex to the plasma membrane. J Neurosci 32:7356-7363

15. Epley KE, Urban JM, Ikenaga T, Ono F (2008) A modified acetylcholine receptor $\delta$-subunit enables a null mutant to survive beyond sexual maturation. J Neurosci 28:13223-13231

16. Charnet P, Labarca C, Lester HA (1992) Structure of the gammaless nicotinic acetylcholine receptor: learning from omission. Mol Pharmacol 41:708-717

17. Meyer A, Van de Peer Y (2005) From $2 R$ to $3 R$ : evidence for a fish-specific genome duplication (FSGD). BioEssays 27:937-945

18. Papke RL, Ono F, Stokes C et al (2012) The nicotinic acetylcholine receptors of zebrafish and an evaluation of pharmacological tools used for their study. Biochem Pharmacol 84:352-365

19. Lefebvre JL et al (2004) Increased neuromuscular activity causes axonal defects and muscular degeneration. Development $131: 2605-2618$

20. Walogorsky M, Mongeon R, Wen H et al (2012) Acetylcholine receptor gating in a zebrafish model for slow-channel syndrome. J Neurosci 32:7941-7948

21. Devoto SH, Melançon E, Eisen JS, Westerfield M (1996) Identification of separate slow and fast muscle precursor cells in vivo, prior to somite formation. Development 122:3371-3380

22. Blanco G, Coulton GR, Biggin A et al (2001) The kyphoscoliosis (ky) mouse is deficient in hypertrophic responses and is caused by a mutation in a novel muscle-specific protein. Hum Mol Genet 10:9-16 
23. Frail DE, McLaughlin LL, Mudd J, Merlie JP (1988) Identification of the mouse muscle 43,000-dalton acetylcholine receptorassociated protein (RAPsyn) by cDNA cloning. J Biol Chem 263:15602-15607

24. Ono F, Shcherbatko A, Higashijima S-I et al (2002) The zebrafish motility mutant twitch once reveals new roles for rapsyn in synaptic function. J Neurosci 22:6491-6498

25. Ohno K, Engel AG, Shen X-M et al (2002) Rapsyn mutations in humans cause endplate acetylcholine-receptor deficiency and myasthenic syndrome. Am J Hum Genet 70:875-885

26. Ono F (2008) An emerging picture of synapse formation: a balance of two opposing pathways. Sci Signal 1:pe3-pe3

27. Burden SJ (1998) The formation of neuromuscular synapses. Genes Dev 12:133-148

28. Burden SJ, DePalma RL, Gottesman GS (1983) Crosslinking of proteins in acetylcholine receptor-rich membranes: association between the beta-subunit and the $43 \mathrm{kd}$ subsynaptic protein. Cell 35:687-692

29. Lee Y, Rudell J, Ferns M (2009) Rapsyn interacts with the muscle acetylcholine receptor via $\alpha$-helical domains in the $\alpha, \beta$, and $\varepsilon$ subunit intracellular loops. Neuroscience 163:222-232

30. Borges LS, Yechikhov S, Lee YI et al (2008) Identification of a motif in the acetylcholine receptor $\beta$ subunit whose phosphorylation regulates rapsyn association and postsynaptic receptor localization. J Neurosci 28:11468-11476

31. Ono F, Higashijima S, Shcherbatko A et al (2001) Paralytic zebrafish lacking acetylcholine receptors fail to localize rapsyn clusters to the synapse. J Neurosci 21:5439-5448

32. Marangi PA, Forsayeth JR, Mittaud P et al (2001) Acetylcholine receptors are required for agrin-induced clustering of postsynaptic proteins. EMBO J 20:7060-7073

33. Séguéla P, Wadiche J, Dineley-Miller K et al (1993) Molecular cloning, functional properties, and distribution of rat brain alpha 7: a nicotinic cation channel highly permeable to calcium. J Neurosci 13:596-604
34. Jennings CG, Dyer SM, Burden SJ (1993) Muscle-specific trkrelated receptor with a kringle domain defines a distinct class of receptor tyrosine kinases. Proc Natl Acad Sci USA 90:2895-2899

35. Okada K, Inoue A, Okada M et al (2006) The muscle protein Dok-7 is essential for neuromuscular synaptogenesis. Science 312:1802-1805

36. Kim N, Stiegler AL, Cameron TO et al (2008) Lrp4 is a receptor for agrin and forms a complex with MuSK. Cell 135:334-342

37. Lefebvre JL, Jing L, Becaficco S et al (2007) Differential requirement for MuSK and dystroglycan in generating patterns of neuromuscular innervation. Proc Natl Acad Sci USA 104: 2483-2488

38. Zhang J, Lefebvre JL, Zhao S, Granato M (2004) Zebrafish unplugged reveals a role for muscle-specific kinase homologs in axonal pathway choice. Nat Neurosci 7:1303-1309

39. Jing L, Lefebvre JL, Gordon LR, Granato M (2009) Wnt signals organize synaptic prepattern and axon guidance through the zebrafish unplugged/MuSK receptor. Neuron 61:721-733

40. Michalk A, Stricker S, Becker J et al (2008) Acetylcholine receptor pathway mutations explain various fetal akinesia deformation sequence disorders. Am J Hum Genet 82:464-476

41. Flanagan-Steet H, Fox MA, Meyer D, Sanes JR (2005) Neuromuscular synapses can form in vivo by incorporation of initially aneural postsynaptic specializations. Development 132:4471-4481

42. Panzer JA, Song Y, Balice-Gordon RJ (2006) In vivo imaging of preferential motor axon outgrowth to and synaptogenesis at prepatterned acetylcholine receptor clusters in embryonic zebrafish skeletal muscle. J Neurosci 26:934-947

43. Kimura Y, Hisano Y, Kawahara A, Higashijima S-I (2014) Efficient generation of knock-in transgenic zebrafish carrying reporter/driver genes by CRISPR/Cas9-mediated genome engineering. Sci Rep 4:6545 